\title{
ADAPTIVe InTElligent ManUfaCturing CONTRol Systems
}

\author{
Sergey A. Yerofeyev, Oleg S. Ipatov, Sergey A. Markov, Vyacheslav V. Potekhin, \\ Angelina S. Sulerova, Viacheslav P. Shkodyrev
}

Peter the Great St. Petersburg Polytechnic University, Polytechnicheskaya 29, 195251 St. Petersburg, Russia

\begin{abstract}
The article is devoted to development of the concept of the integrated intelligent control system for complex manufacturing process. The base of the system is recurrent decomposition method, which allows reconfiguring systems logical structure in case of the given optimum criteria. A recurrent decomposition model of the general problem of manufacturing process automation and the algorithm of multi-level control for automated facilities with scalable tree structures are suggested.

In this paper, the design of processes, as well as the problem of automating the lifecycle of the product are considered within the concept of Industry 4.0. The methods of final product quality control based on the control and testing processes optimization are considered as well as methods of structural and parametric optimization of technological processes. Some application examples of the developed mathematical models for technological systems are given.
\end{abstract}

Keywords: Intelligent control systems; manufacturing process; adaptive structure; automation; structural and parametric optimisation; process model; recurrent decomposition.
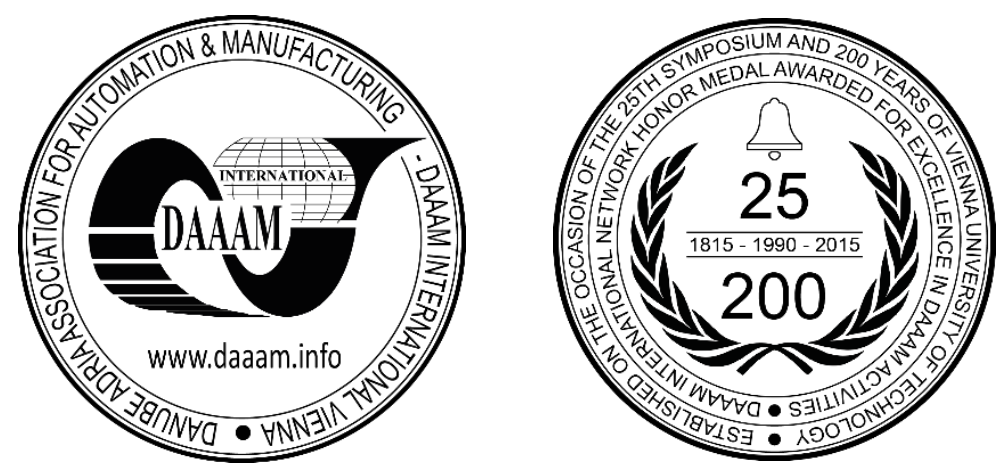

This Publication has to be referred as: Yerofeyev, S[ergey]; Ipatov, O[leg]; Markov, S[ergey]; Potekhin, V[yacheslav]; Sulerova, A[ngelina] \& Shkodyrev, V[iacheslav] (2016). Adaptive Intelligent Manufacturing Control Systems, Proceedings of the 26th DAAAM International Symposium, pp.1016-1024, B. Katalinic (Ed.), Published by DAAAM International, ISBN 978-3-902734-07-5, ISSN 1726-9679, Vienna, Austria

DOI: $10.2507 / 26$ th.daaam.proceedings.143 


\section{Introduction}

Technological expediency, operational necessity and economic efficiency allowed wide spreading of industrial automation. The fourth industrial revolution, which is also called "Industry 4.0", combines all technological equipment in the manufacturing process with information networks. This completely new architecture of industrial can be implemented not only in new companies but also by stages expand on existing ones. Experience in the implementation of such systems already have companies such as Festo and Siemens, but our goal was to develop a methodology for the integrated automation of small-scale production.

All-round automation of complex manufacturing process includes its fuzzy model to be created [1]. Afterwards this model is to be refined step by step. Initial uncertainty may be caused by variable scheme of process stepping into separate places with possible arising of new automated working places (AWP), variable list or specifications of actions performed at such places. Firstly created architecture undergoes multiple changes during developing. The reason of such change may be criterion to determine the optimum functional considering the targets which must be reached within manufacturing process. Final purpose of iterative refining of automation scheme is to detail the target and functional specifications of technology process down to indivisible elements (atoms).

Above described features of automation scheme allow to apply the method of recurrent decomposition [2]. Following this method there is created the tree [3] of tasks to be performed in accordance with stated targets. The description is provided by variability which is necessary to subsequently adapt the scheme to refined requirements. Such variability is ensured by creating the aggregates (equivalence classes) of tasks with defined common properties. This target model is also called as model of problem domain. Moreover, there is created the tree module view of functionalities making possible to solve stated tasks. This tree called as functional model is provided by variability which afterwards will be reduced to the specific way of realization. In particular one functional module may give possibility to select between different types of regulator following the synthesis of optimal control system. In practice the strategy requires to create the model of actions for the control system based on the sets of selected generalized algorithms.

Considered target and functional models of process automation are created following the formal language and to be repeatedly refined. During project realizing the collective views of production places and corresponding realization modules are replaced by its more detail views taking down to the indivisible elements layer of representation. The advantage of such recurrent method application is the switching to the formal style of problem description with possibility to reconfigure the structure and some variability of its nodes.

As result of this method application the scope of control system actions described in the functional model covers all tasks of automation system which are indicated in the target model and all models are reduced to indivisible nodes characterized by some parameters. Selection between multiple algorithmic routes requires to fix the values of such parameters or determine them automatically in accordance with set performance criterions. The complexity of such optimization is increased down to top following the tree structure, starting from indivisible nodes at the bottom layer. Therefore there may be useful to apply mathematical models in conjunction with empirical knowledge which for example may be taken from customer during project realizing. Such combination of formalism and adaptability allows to determine the final rote on the base of created models as soon as possible.

\section{Concept of the Industry 4.0}

Industry 4.0 is based on cyber physical systems providing M2M connectivity, planning and adaptation of personal behavior according to ambient conditions, learning of new models and ways of behavior and interpreting information about the environment [4-5]. It provides an effective release of even minimal batches with rapid amendments to products and multi-variant [6-8].

Figure 1 shows the levels of the Industry 4.0 concept. The automated control and monitoring system is designed as a part of production automation project for performance improvement and increase of economic efficiency of the production line. Schematic diagram of APCS is shown in Fig. 2.

The complete system has a hierarchic structure where the lower level, the PLC level, is responsible for acquisition and transmission of the information about objects and parameters of the technological process to the upper levels. Communication with the equipment recording, monitoring the readings and directly controlling the objects is provided by PLC (programmable logic controller). All sensors and execution units of the equipment involved in the technological process are connected to the PLC [9].

The Industry 4.0 concept requires the PC (Plant Connectivity) element for communication between the main software portion of APCS and the equipment implementing the technological process, and the data transmission server may be used for this purpose.

The upper level is used to set the general control algorithm and to arrange the high-level interface with the system users (productions engineers and service staff), it consists of AWP (automated work place) and a server providing the control and information acquisition process. 


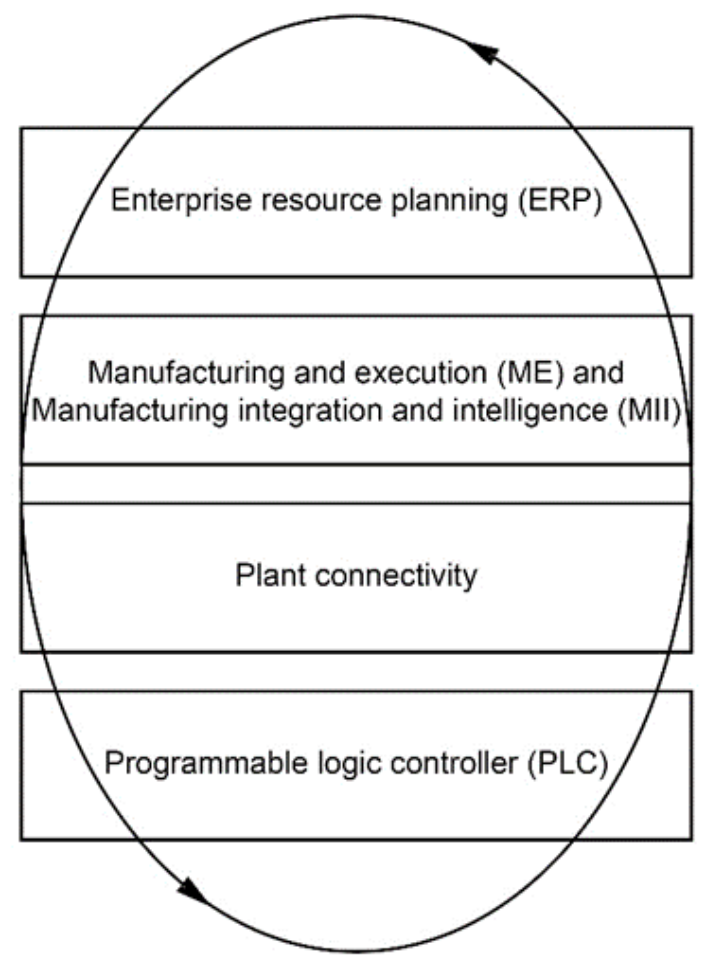

Fig. 1. Schematic diagram of the Industry 4.0 concept.

The server portion of APCS consists of several components: a web-server, an application server, a database server, a print server. The database server provides long-term storage and acquisition of production data. The application server executes the business logic related to the manufacturing processes and control processes (control algorithm), the web-server is the basis of the AWP operation.

The server portion contacts the PLC, the equipment is also connected. The upper level MES subsystem functions are performed by the application server which, in turn, solves the tasks of synchronization, coordination, analysis and optimization of the product release within a certain production on a real time basis.

To coordinate the data with the external source, the thin client building principle using the web-server may be implemented.

\section{Method of structural algorithmic synthesis of hardware and software solutions for the manufacturing processes adaptive control}

The method is developed intended for flexible adaptive control of the complex manufacturing processes when manufacturing program as well as technological processes, deviation of critical parameters and other ambient conditions could be changed during the process $[10,11]$.

In this paper only discrete sequential manufacturing processes which characterized by structured production areas with certain technological operations are concerned.

Real necessity in flexible adaptive control systems becomes apparent with multifunction (robotized) production areas when several technological operations could be carried out at one production area and when one technological operation could be carried out at different production areas.

Such a developed hierarchical structures allow technological sequences design by many different ways, which could be interrelated and limited by each other but carried out at the same time to realize some optimum solution for the given cost function with known resources and technology restrictions.

The method developed could be implemented as an extension of distributed control system (DCS) at manufactures with high automatization level. But also it gives result as a source of management instructions for the manufactures with low automatization level. Technical realization of the method implementation result in hardwaresoftware complex (HSC) being adaptive and optimization control element (AOC) in the whole control systems structure [12].

For the initialization of the AOC procedures below should be carried out:

1. Production areas description

a. technological operations description (input/output details and components, instruments, operation time, bill of materials etc.) 


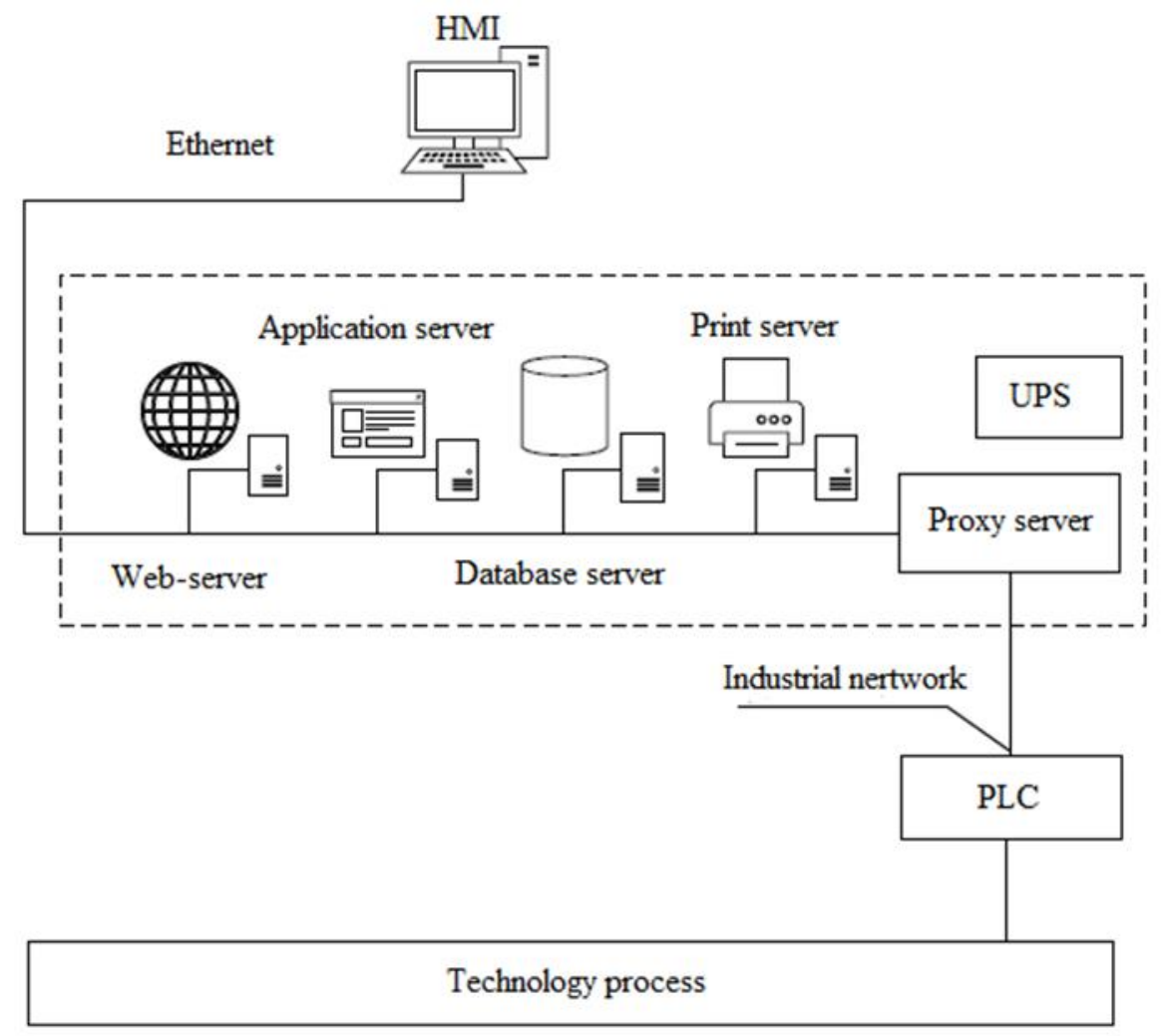

Fig. 2. Schematic diagram of APCS.

b. operation transition time and resources

c. hardware and software for operations

d. operator instructions

2. Technology operations sequences design for all products manufactured

3. Manufacturing program generating

a. order information and identification

b. product types, quantities, production rates

c. product quality requirements

d. product test requirements

e. resources restrictions

f. cost function (minimal prime cost, minimal manufacturing time, minimal resources, etc.)

For the next step AOC routines in real time scale with algorithm below realization:

1. All possible production sequences synthesis for every product manufacturing

2. Variation parameters determination for every production area

3. Cost function problem solution for given time interval (shift, day, month...)

4. Optimal parameters set generation

5. Optimal set of production sequences with set points generating for the manufacturing program

6. Production program for every production area generating

7. Algorithm for operation procedures for every production area synthesis

8. Request generating for hardware, software, components, resources, etc. for every production area;

9. Direct model calculation for controlled and registered technological and production parameters limitations;

10. Registering and control of the technological and production parameters.

If some events which could change parameters of manufacturing program is registered during the process then AOC initializes new optimization algorithm routine for the rest production time interval, then find new optimal set of production sequences and correct production programs for every production area.

Such events could be:

- Manufacturing program modification;

- Technology operation sequences modification; 
- Manufacturing parameters out of limits.

The realization of the method developed gives an excellent result for different industrial implementations as a part of manufacturing execution system (MES) [13] and leads to:

- $\quad$ wide product list manufacturing;

- flexible in-time manufacturing process modification;

- $\quad$ productivity increase;

- low prime cost.

\section{Mathematical model}

One the most complicated issues in production engineering is solving the task of creating an optimal route for production of parts, assembly units and the item in general considering the equipment loading, its capabilities and condition.

The system designing begins with the synthesis of the initial variant of its structure, preliminary definition of content of the technological operations by combining operations (transitions) in them as well as general sequence of the formed operations execution with the required corrections in their content. A mathematical model is created to evaluate this variant.

The technological solution with the selected physical principle of operation is searched for according to the structural synthesis method.

Figure 3 shows an example of the technological layout of the phase shifter assembly line. Definition of optimal parameter values of the technical system elements with the known structure is the task of parametric synthesis or parametric optimization.

Network diagram of the manufacturing process allows to show the technological dependence and sequence of the work package execution coordinating the schedules considering resources spent and the work cost indicating critical points [14]. Figure 4 shows the sequence of technological operations of the phase shifter assembly process where $\mathrm{S}$ is the object status corresponding with the technological process at a certain moment, $\mathrm{A}$ is the part movement to the next position/next module.

The solution of the structural optimization tasks may be based on presentation of the structure as diagrams, comparative analysis of structures based on the limited amount of structural parameters, however, selection of the optimal variant of the designed complex technical object structure with the methods based on the exhaustive search is a rather time-consuming procedure.

Using the mathematical programming methods to solve the tasks of the structural synthesis of technical objects requires a lot of preliminary preparation for solution area study, and it is not always feasible due to the difficulty of consideration of multiple factors impacting the correctness of the task of optimal designing and due to significant calculation difficulties in solving the tasks of mathematical programming of high dimension.

Methods of parametric optimization of technological processes are based on standard patterns solving mathematical programming tasks: linear, non-linear, convex and integer-valued [15].

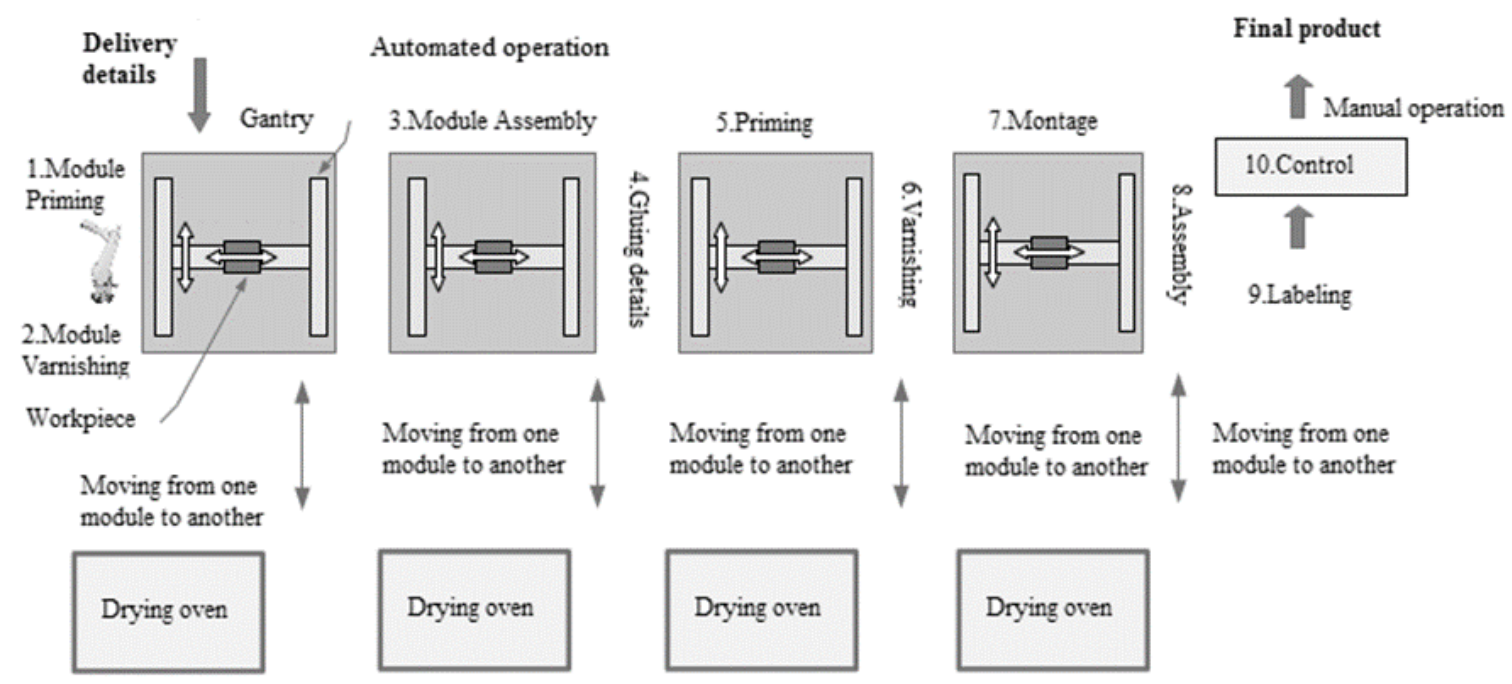

Fig. 3. Technological layout of the phase shifter assembly line. 
The total time of a batch assembly $t(T)$ according to the technological process $T$ was used as the optimization criterion during technological design of the phase shifter assembly process. The optimal $T_{\text {opt }}$ variant is the variant of the technological process with the minimal t value:

$$
t\left(T_{\text {opt }}\right)=\min t(T)
$$

In general, when technological processes are being optimized, the optimization search methods are used for they do not impose severe restrictions on optimization criteria and solution existence domain. The principle of the optimization method is to find the sequence of the technological process variants:

$T_{1} \ldots T_{i} \ldots T_{n}$

where each subsequent variant is more preferable than the previous one, i.e. $C\left(T_{i}\right)>C\left(T_{i+1}\right)$. At the extreme, the mentioned sequence must converge to a sufficiently small solution area, i.e. the variant close to optimal.

Below is an example of a mathematical model of the phase shifter assembly process allowing to make all necessary calculation for the parametric optimization where the optimization parameter is the total time of a batch assembly.

$$
\begin{aligned}
& t_{j}(n)=\sim+(n-1) t_{\max } \\
& \sim_{t}=\sum_{i} t_{i} \\
& t_{\max }=\max \left\{t_{i}\right\} \\
& T=\sum_{j} t_{j}(n)+\sum_{f} t_{f}^{*}
\end{aligned}
$$

where $T$ is the total time of a batch assembly, $n$ is the number of parts in a batch, $t_{j}$ is the time required for a batch assembly before the drying process starts, $\tilde{t} t$ is the time required for one part treatment before the drying process starts, $t_{i}$ is the time required for the technological operation $i, k$ is the total amount of technological operations before the drying process, $\mathrm{t}_{\mathrm{f}}^{*}$ is the time required for the drying process number $f$, optimization may also be based on resource intensity, power consumption, accuracy, floor space.

In the market conditions of economic management, searching for the optimal variant of solving the task related to creation of the production program of an enterprise is of great economic importance. Definition of optimal plans with the contemporary models and mathematical methods is one of the most important research areas and the subsequent use of the internal resources and the increase of the economically efficient work of the enterprise on this basis. The result of the enterprise plan (annual, quarterly, semi-annual) creation is the establishment of the set of products to be manufactured and the amount of their release as well as providing achievement of one or several goals (maximization of the gross profit, maximization of the amount of the sold products, maximization of the cultivated target market share, minimization of the total original cost of the programmed output, etc.).

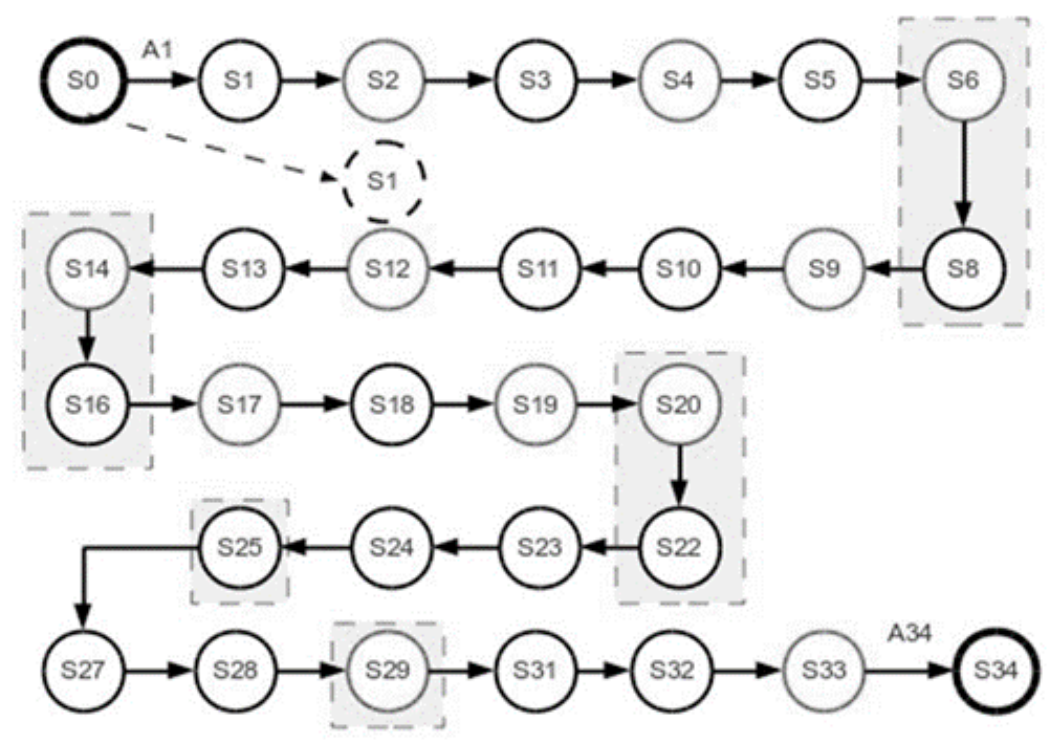

Fig. 4. Technological sequence of the phase shifter assembly process. 


\section{Example of the process}

Below shows the example of the synthesis of optimal variant of the structure of the process when the need to automate some operations. Figure 5 illustrates a process sequence casting of high pressure.

The result of the audit the current status of manufacturing was to study the characteristics of the control object and the construction of conceptual, network, permutation and table models, see Fig.6.

Multivariance technological routes can be represented as a directed weighted graph, technical solutions which differ from one another composition, the amount of equipment, the cost and time of execution the relevant operation. Let us assume that there are many technological routes:

$$
\begin{aligned}
& M=\left\{M_{j}\right\} ; \\
& |M|=k, m_{j}=\left\{o_{j i}\right\} ; \\
& \left|m_{j}\right|=d_{j}
\end{aligned}
$$

where $o_{j i}-$ technological operations

$$
\begin{aligned}
& K=\left\{k_{f}\right\} \\
& |K|=p ; \\
& k_{f}=v_{f g} \\
& \left|k_{f}\right|=a
\end{aligned}
$$

where $k_{f}$ - types of equipment, $v_{f g}$ - models of equipment.

Restrictions:

$$
\begin{aligned}
& R_{f g}>0, C>0, T_{f g}>0 ; \\
& n>2, N>0, w_{i}>0 ; \\
& C_{f g} \leq N, T_{f g} \leq T_{t} \\
& \sum_{i=1}^{n} w_{i}=1
\end{aligned}
$$

where $C_{f g}$ - model equipment cost, $R_{f g}$ - resource consumption, $T_{f g}$ - technological operations runtime, $T_{t}$-total operation time per detail, $N$ - cash fund.

$$
\begin{aligned}
& g\left(m_{j}\right)=\sum_{i=1}^{n} w_{i} f_{i}\left(m_{j}\right) ; \\
& w_{i} \geq 0, \sum_{i=1}^{n} w_{i}=1, i=1, \ldots, n ; m_{j} \in M
\end{aligned}
$$

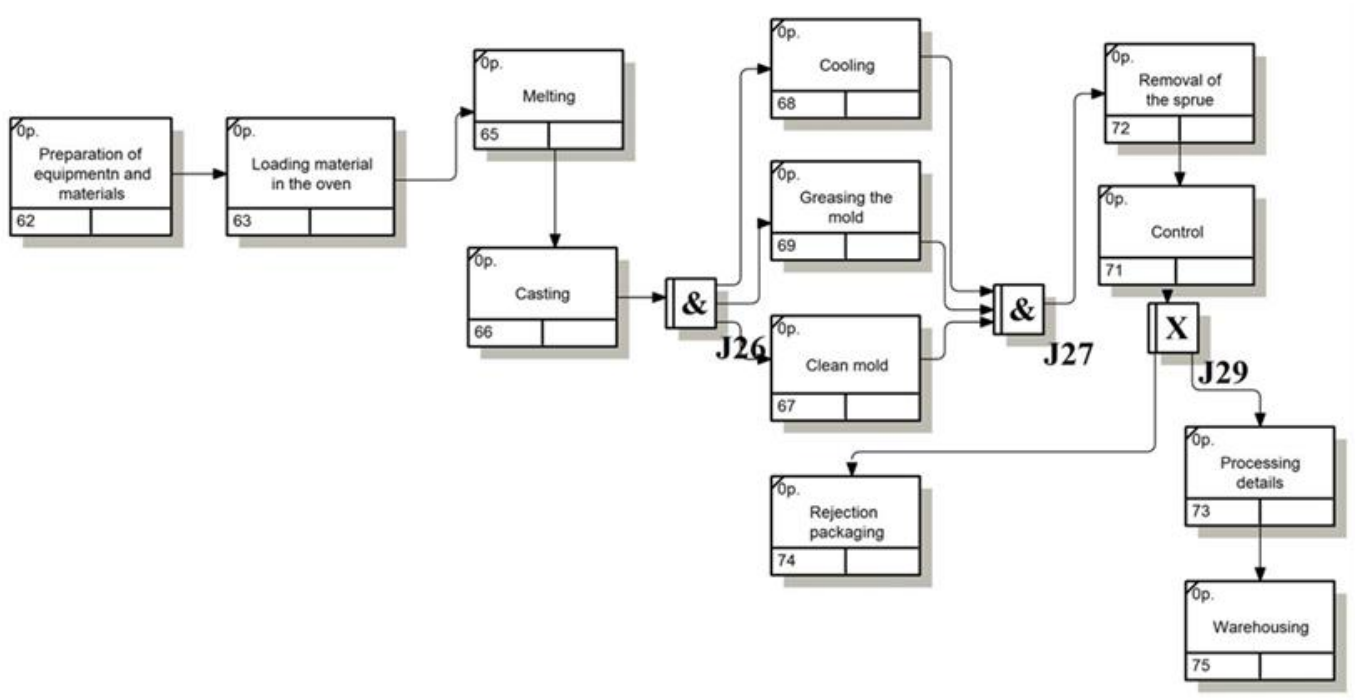

Fig. 5. Diagram of the process casting under pressure parts.

A path connecting the initial and final vertices of the technical solutions is to find that the total path length is minimal in view of the restrictions imposed.

In this project one-criteria and multi-criteria problems were solved by using Dijkstra's algorithm, selected on the basis of comparative analysis of the search engine optimization techniques in a task. 
The selected design solution is calculated techno-economic indicators in accordance with the production program. In case of unsatisfactory results of adjustment the complex structure modification is required.

a

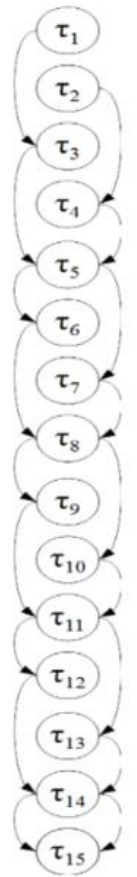

b
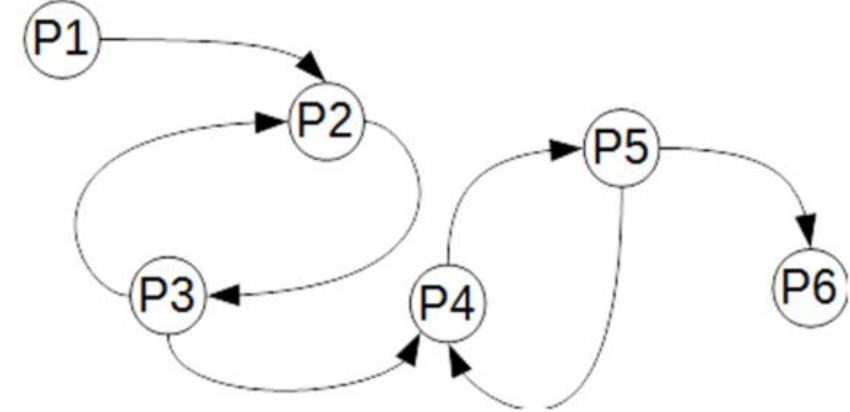

c

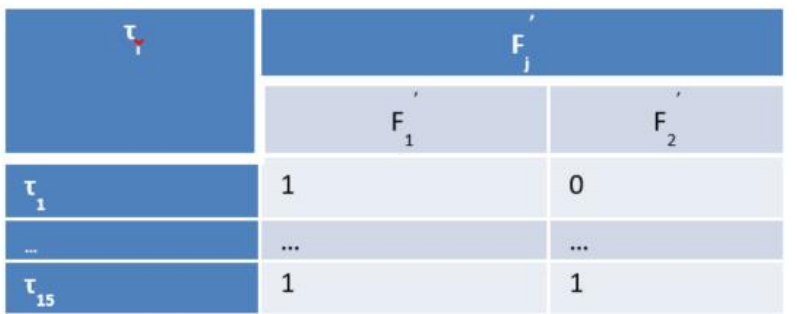

Fig. 6. (a) Network model; (b) permutation model; (c) table model, where $P_{i}$ - technology department, $\tau_{i}$ - technological operations, $F_{j}^{\prime}$ - detail.

\section{Conclusion}

Using the approach is considered a recurrent model were developed and tested models of flexible intelligent manufacturing technological processes which advantages are described in [16] were created within this project.

Designed control systems based on Industry 4.0 principles. In the article principles of constructing intelligent control system are shown on different levels.

Analysis and later structural and parametric optimisations with aspects shown in the provided mathematical models are the next step to be done to improve existed technological processes. The merits of the developed concept are increased production flexibility and mobility, reduced time of the production cycle. General control functions integrated in a single user interface and interaction of the lower and upper levels with a data transmission server and application server allow to minimize the intervention of the service staff to control the technological process, to record the system events in order to analyze them and involve the operator in due time, if necessary. The integrated industry development will allow to exceed the scope of a certain production and to use the infrastructure and data of several enterprises altogether.

\section{Acknowledgements}

The article is published in the framework of the project TEMPUS 544490-Tempus-1-2013-1-ES-TEMPUSJPCR and describes the part of the project conducted by SPbPU.

\section{References}

[1] Fedorov A., Shkodyrev V., Zobnin S. «Knowlrdge based planning framework for intelligent distributed manufacturing systems».Lecture notes in computer science (incliding subseries Lecture Notes in Artificial intelligence and Lecture Notes in Bioinformatics), volume 9141, 2015, pp. 300-307

[2] A.V. Masloboev, V.A. Putilova, A.V. Sioutinea. Multilevel recurrent model for hierarchical control of complex regional security. Scientific and Technical Journal of Information Technologies, Mechanics and Optics, 2014 , № 6 (94), pp. 163-170.

[3] Mesarovic M.D., Macko D., Takahara Y. Theory of Hierarchical Multilevel Systems. NY-London: Academic Press, 1970. p.294. 
[4] Wen-Tsuen Chen, Youn-Long Lin, Chen-Yi Lee, Jeng-Long Chiang, Meng-Fan Chang, Shih-Chieh Chang. «Strengthening modern electronics industry through the National Program for intelligent electronics in Taiwan», Access, IEEE, volume 1. 2013. pp.123-130

[5] T. Kushida, G.S. Pingali. «Industry cloud - effective adoption of cloud computing for industry solutions». Cloud computing (CLOUD), 2014 IEEE 7th International Conference on, 2014 , pp. 753-760.

[6] Prof. Dr. Henning Kagermann, Prof. Dr. Wahlster, Dr. Johannes Helbig. Final report of the Industrie 4.0 Working Group «Recommendations for implementing the strategic initiative Industrie 4.0», 2013. p.80.

[7] Haskovic, D., Katalinic, B., Kukushkin, I. Role o. The Adviser Module i. The hybrid assembly subordinating control structure, Proceedings of DAAAM International Symposium on Intelligent Manufacturing and Automation, DAAAM 2014; Vienna; Austria; 26 November 2014 through 29 November 2014, Volume 100, Issue C, 2015, Pages 1706-1713.

[8] Gastermann, B., Stopper, M., Kossik, A., Katalinic, B. Secure implementation of an on-premises cloud storage service for small and medium-sized enterprises, Proceedings of DAAAM International Symposium on Intelligent Manufacturing and Automation, DAAAM 2014; Vienna, Austria; 26 November 2014 through 29 November 2014, Volume 100, Issue C, 2015, Pages 574-583.

[9] «PLCs and ERP/APS+MES+ACS integration», Rational Enterprise Management — information and analytical magazine for IT managers and experts of industrial enterprises, research and design organizations, 2009, No.2, pp. $50-51$.

[10] Kovalevsky V.E., Potekhin V.V., Shkodyrev V.P., Arseniev D.G. Smart Manufacturing with Distributed Knowledge-Base Control Networks. 2014. AST Symposium on Automated Systems and Technologies. p.85

[11] Vyacheslav Shkodyrev, Aleksandr Fedorov. Knowledge-based automation in smart manufacturing systems. Proceedings of the Symposium Automated Systems and Technologies. St. Petersburg, Russia. 2015.

[12] I.G.Chernorutski, Optimization methods in Control Theory. PITER. 2004. (in Russian)

[13] Heiko Meier, Franz Fuchs, Klaus Thiel. Manufacturing Execution Systems. Optimal Design, Planning and Deployment. 2009 McGraw-Hill Companies, Inc.

[14] S. A. Polyakov. Mathematical models and engineering production facilities modeling: textbook - M.: Moscow state university publisher, 2011. p.104.

[15] A. Y. Krukov. Mathematical modeling of processes in mechanical engineering. - Perm: Perm State Technical University Publisher, 2007. p.322.

[16] V. Y. Shishmarev. Automation of production processes in mechanical engineering: textbook. - M.: Academy, 2007. p.368. 